\title{
Planet heating prevents inward migration of planetary
}

\section{cores}

\author{
Pablo Benítez-Llambay ${ }^{1}$, Frédéric Masset ${ }^{2}$, Gloria Koenigsberger $^{2}$, and Judit Szulágyi ${ }^{3}$
}

March 27, 2018

${ }^{1}$ IATE, Observatorio Astronómico, Universidad Nacional de Córdoba, Laprida 854, Córdoba, X5000BGR, Argentina

${ }^{2}$ Instituto de Ciencias Físicas, Universidad Nacional Autónoma de México, Av. Universidad s/n, 62210 Cuernavaca, Morelos, Mexico

${ }^{3}$ University of Nice-Sophia Antipolis, CNRS, Observatoire de la Côte d'Azur, Laboratoire Lagrange, F-06304, Nice, France

Planetary systems are born in the disks of gas, dust and rocky fragments that surround newly formed stars. Solid content assembles into ever-larger rocky fragments that eventually become planetary embryos. These then continue their growth by accreting leftover material in the disc. Concurrently, tidal effects in the disc cause a radial drift in the embryo orbits, a process known as migration ${ }^{1+4}$. Fast inward migration is predicted by theory for embryos smaller than three to five Earth masses ${ }^{5+7}$. With only inward migration, these embryos can 
only rarely become giant planets located at Earth's distance from the Sun and beyond ${ }^{899}$, in contrast with observations ${ }^{10}$. Here we report that asymmetries in the temperature rise associated with accreting infalling material ${ }^{11 / 12}$ produce a force (which gives rise to an effect that we call "heating torque") that counteracts inward migration. This provides a channel for the formation of giant planets ${ }^{8}$ and also explains the strong planet-metallicity correlation found between the incidence of giant planets and the heavy-element abundance of the host stars 13,14 .

We solve the equations governing the disc hydrodynamics in combination with the equations of radiative transfer. Planets have an angular momentum that increases with their orbital radius. In the case of a nearly circular orbit, the rate of change of angular momentum, or torque, gives the migration rate. Our calculations are performed in three dimensions, yielding a reliable value for the net torque, from which the direction and rate of migration are inferred.

Our fiducial computation is one in which a rocky core with 3 Earth masses is located at a distance comparable to that of Jupiter from the Sun and is being bombarded by solid material at a rate that doubles its mass in 100 thousand years. We assume that the gravitational energy of the infalling solid material is transformed entirely into heat and ultimately radiated by the planet ${ }^{11}$. A second computation is performed with the same set up, but without the planet's radiation, in order to distinguish the effects of the heating torque from other torques. We find that the heating torque (defined as the torque difference between cases with accretion turned respectively on and off) has a positive sign (figure 1), which enables it to counteract the effect of the standard, negative torque. The latter includes all torque components of the non-heating case, and is always negative for small 
mass embryos (typically smaller than $5 M_{\oplus}$, where $M_{\oplus}$ is the Earth's mass). Thus, the effect of the heating torque is to either slow down the inward migration, cancel it, or reverse its direction. The most important factors governing the strength of the heating torque and thus, the direction of migration, are the accretion rate of the embryo, its mass and the opacity of the disc. For our fiducial values of opacity, disc structure and embryo mass, we find that outward migration occurs for accretion rates corresponding to a mass doubling time less than approximately 60 thousand years. For larger mass doubling times (i.e., smaller accretion rates), the heating torque can at best slow down the inward migration but not reverse it (figure 2).

The heating torque has a large efficiency over the mass interval $0.5-3 M_{\oplus}$, which is precisely the range of masses where counteracting inward migration is required in order to allow further embryo growth at distances where giant planets are expected to form 9 . Masses smaller than $0.5 M_{\oplus}$, for which the heating torque has a lower efficiency, migrate inward only a negligible fraction of their orbital radius by the time they double their mass.

Some insight into the physics of our new torque component can be gained by examination of a mass density map in the planet vicinity. The energy released by the planet heats the optically thick disc in its vicinity. As the latter maintains its pressure equilibrium, hotter regions are less dense than the surrounding ambient material. As a consequence of the flow pattern around the planet, two under-dense lobes appear, one leading and one following the planet, when the heating is included in the calculation (figure 3). Protoplanetary discs rotate in general at a speed slightly smaller than the Keplerian speed, and the corotation (the location at which the material orbits the star at same pace as the planet) lies slightly toward its star from the planet. This situation favours the lobe that 
appears behind the planet: its material approaches closer to the planet, receives more heat and is consequently less dense than the other lobe, leading to a positive torque on the planet. We explored the dependence of the heating torque on the gradient of surface density (which leads to the shift between the planet and its corotation), and we found that the heating torque does indeed scale with the distance to corotation. Only in the non-realistic cases in which the corotation is further out than the planet (when the gas pressure increases outward) do we find a negative heating torque. The heating torque therefore constitutes a robust trap against inward migration in any realistic disc, when accretion rates are large enough.

In the limit of a very large accretion rate, the heating torque largely dominates other torque components, and the net torque is directly proportional to the accretion rate. Since the migration rate is proportional to the torque, it follows that the final distance over which an embryo migrates is a function of the mass it accretes. We find that an embryo initially smaller than the Earth would at most double its semi-major axis by the time it reaches 5 Earth masses.

The finding that the heating torque can produce outward migration for accreting embryos in their earliest stage of development has implications for the formation of planetary systems in general, and the Solar System in particular. It opens a new route for the formation of gas giant planets and alleviates the problem encountered by current models of planetary population synthesis which predict too many super-Earths ${ }^{9}$ and a low yield of giant planets. The general picture that now emerges is that embryos with masses in the range $0.3-5$ Earth masses are able to avoid inward migration when accretion rates are large. By the time the heating torque efficiency drops, they have entered a regime in which other mechanisms driving outward migration come into play ${ }^{5|9| 15}$. 
Embryos that are formed when accretion rates are low will still undergo inward migration but at a slower rate. The overall migration behaviour therefore displays a bifurcation depending on the accretion rate of solids.

This bifurcation provides a simple and natural explanation for the very strong correlation found between the incidence of giant planets and the heavy-element content (i.e., metallicity) of the host $\operatorname{star}^{13} \mid 14$. That is, since the heating torque scales with the accretion rate and the accretion rate, in turn, scales with the amount of solid content (a proxy of which is the metallicity), protoplanetary discs with larger metallicity will engender planets that can avoid inward migration and grow to become giant planets. In contrast, embryos born in lower metallicity environments cannot avoid inward migration, leading to results as hitherto found in models of planetary population synthesis, with low yields of giant planets and ubiquitous super-Earths. These might therefore be more abundant in metal-poor systems, as suggested by a recent planet search with accurate stellar metallicities ${ }^{16}$. The incidence of super-Earths with the metallicity of the host star is however debated ${ }^{14}$. Recent models have contemplated the in situ formation of super-Earths at small orbital distances from the $\operatorname{star}^{17} \mid 18$ ( $\lesssim 1 \mathrm{AU}$, one AU being the average distance from the Earth to the Sun). We have not performed calculations for embryos so close to the star, where the disk parameters are still poorly constrained, but we note that the very short mass doubling times inferred for some planets very close to their star ${ }^{18}$ would probably yield an extremely large heating torque, the magnitude of which remains to be properly calculated to assess the likelihood of in situ formation scenarios. The implications for the formation of our own Solar System are somewhat more speculative, but are important to highlight. The parameters of our fiducial run are typical of those found beyond 
the snow line (the distance at which water ice condenses). On the warm side of the snow line we expect the heating torque to have a much reduced efficiency, because the bombardment rate and the disc opacity drop substantially. With a heating torque producing outward migration, we expect all embryos having formed beyond the ultimate location of the snow line to have experienced a sizable outward migration, thus causing a large depletion of solid material in this region. Hence, a prediction of our torque mechanism is that a depleted region should be present inside the orbit of the first giant planet in many planetary systems. In our Solar System, such a region may correspond to the asteroid belt, which only contains approximately 0.001 Earth mass of solid material ${ }^{19}$. Within our framework, Jupiter's rocky core could have been assembled from embryos originating from this region.

A priori, the heating torque would not have been expected to have the same order of magnitude as the tidal torque. It depends primarily on the planet's physical radius, dust opacity and mass accretion rate, whereas the tidal torque depends on the gaseous disc's surface density and temperature. The serendipitous coincidence of both torques is remarkable and allows the bifurcation between inward and outward migration to occur for accretion rates that lie within the range of the largest and smallest accretion rates believed to prevail in protoplanetary systems.

Although further implications of this new torque component can only be derived from a fine-tuned analysis of all parameters of the disc and the embryo, it is clear that the heating torque provides a unifying mechanism for gaining a deeper understanding of giant planet formation in general and the specifics which gave rise to our own system. 


\section{References}

[1] P. Goldreich and S. Tremaine. Disk-satellite interactions. Astrophys. J., 241:425-441, 1980.

[2] W. R. Ward. Protoplanet Migration by Nebula Tides. Icarus, 126:261-281, 1997.

[3] W. Kley and R. P. Nelson. Planet-Disk Interaction and Orbital Evolution. Ann. Rev. Astron. Astrophys., 50:211-249, 2012.

[4] C. Baruteau, A. Crida, S.-J. Paardekooper, F. Masset, J. Guilet, B. Bitsch, R. P. Nelson, W. Kley, and J. C. B. Papaloizou. Planet-Disc Interactions and Early Evolution of Planetary Systems. Preprint at http://arxiv.org/abs/1312.4293, 2013.

[5] S.-J. Paardekooper, C. Baruteau, and W. Kley. A torque formula for non-isothermal Type I planetary migration - II. Effects of diffusion. Mon. Not. R. Astron. Soc., 410:293-303, 2011.

[6] F. S. Masset and J. Casoli. Saturated Torque Formula for Planetary Migration in Viscous Disks with Thermal Diffusion: Recipe for Protoplanet Population Synthesis. Astrophys. J., 723:1393-1417, 2010.

[7] H. Tanaka, T. Takeuchi, and W. R. Ward. Three-Dimensional Interaction between a Planet and an Isothermal Gaseous Disk. I. Corotation and Lindblad Torques and Planet Migration. Astrophys. J., 565:1257-1274, 2002.

[8] H. F. Levison, E. Thommes, and M. J. Duncan. Modeling the Formation of Giant Planet Cores. I. Evaluating Key Processes. Astron. J., 139:1297-1314, 2010. 
[9] C. Cossou, S. N. Raymond, F. Hersant, and A. Pierens. Hot super-Earths and giant planet cores from different migration histories. Astron. Astrophys., 569:A56, 2014.

[10] A. W. Howard. Observed Properties of Extrasolar Planets. Science, 340:572-576, 2013.

[11] J. B. Pollack, O. Hubickyj, P. Bodenheimer, J. J. Lissauer, M. Podolak, and Y. Greenzweig. Formation of the Giant Planets by Concurrent Accretion of Solids and Gas. Icarus, 124:6285, 1996.

[12] C. Mordasini, P. Mollière, K.-M. Dittkrist, S. Jin, and Y. Alibert. Global Models of Planet Formation and Evolution. Preprint at http://arxiv.org/abs/1406.5604, 2014.

[13] D. A. Fischer and J. Valenti. The Planet-Metallicity Correlation. Astrophys. J., 622:1102$1117,2005$.

[14] J. Wang and D. A. Fischer. Revealing a Universal Planet-Metallicity Correlation for Planets of Different Solar-Type Stars. Astron. J., 149:14, 2015.

[15] B. Bitsch, A. Crida, A. Morbidelli, W. Kley, and I. Dobbs-Dixon. Stellar irradiated discs and implications on migration of embedded planets. I. Equilibrium discs. Astron. Astrophys., 549:A124, 2013.

[16] J. S. Jenkins, H. R. A. Jones, P. Rojo, M. Tuomi, M. I. Jones, F. Murgas, J. R. Barnes, Y. Pavlenko, O. Ivanyuk, A. Jordán, A. C. Day-Jones, M.-T. Ruiz, and D. J. Pinfield. Status of the Calan-Hertfordshire Extrasolar Planet Search. In European Physical Journal Web of Conferences, volume 47 of European Physical Journal Web of Conferences, page 5001, 2013. 
[17] B. M. S. Hansen and N. Murray. Testing in Situ Assembly with the Kepler Planet Candidate Sample. Astrophys. J., 775:53, 2013.

[18] E. Chiang and G. Laughlin. The minimum-mass extrasolar nebula: in situ formation of closein super-Earths. Mon. Not. R. Astron. Soc., 431:3444-3455, 2013.

[19] K. J. Walsh, A. Morbidelli, S. N. Raymond, D. P. O’Brien, and A. M. Mandell. A low mass for Mars from Jupiter's early gas-driven migration. Nature, 475:206-209, 2011.

\section{Acknowledgements}

The authors wish to thank A. Morbidelli for a critical reading of a first version of this manuscript.

P.B.Ll. thanks CONICET for financial support. This research was supported by UNAM grants PAPIIT IA101113 and IN105313 and by CONACyT grants 178377 and 129343. J.Sz. acknowledges support from the Capital Fund Management's J.P. Aguilar Grant. The authors wish to thank Ulises Amaya, Reyes García and Jérôme Verleyen for their assistance in setting up the GPU cluster on which the calculations presented here have been run.

\section{Contributions}

P.B.Ll. performed the numerical simulations and their subsequent reduction. F.M. designed the project and wrote the Methods section. G.K. wrote the Main section. J.Sz. provided assistance with the radiative transfer module. All authors contributed to the discussion presented in this manuscript.

\section{Competing financial interests}

The authors declare no competing financial interests. 


\section{Corresponding author}

Correspondence to: Frédéric Masset.

\section{Methods}

\section{Code and numerical method}

We use the publicly available hydrocode FARGO3D (http://fargo.in2p3.fr) to solve the hydrodynamics and radiative transfer equations on a spherical mesh, spanning in azimuth the whole range $[-\pi, \pi]$, in radius the range $[a / 2,(3 / 2) a]$ (where $a$ is the planet's semi-major axis), and in colatitude the range $[\pi / 2-3 h, \pi / 2]$ (where $h=H / r$ is the aspect ratio of the disc). The governing equations of the hydrodynamics module are the continuity equation, the Navier-Stokes equations including all components of the viscous stress tensor, and the energy equation. The equation of state of ideal gases is used. At each hydrodynamical time step, in addition, we solve the radiative energy on the mesh, using flux limited diffusion and a two temperature approach in the grey approximation, and we solve the coupling between thermal and radiative energies $\frac{15}{\text {. These }}$ techniques are standard in the context of protoplanetary disks, in which they allow a simultaneous description of hydrodynamical and radiative effects at reasonable computational $\operatorname{cost}^{20}$.

\section{Fiducial calculation}

The parameters of our fiducial run are:

- A constant opacity $\kappa=1 \mathrm{~cm}^{2} \mathrm{~g}^{-1}$

- A kinematic viscosity $\nu=10^{15} \mathrm{~cm}^{2} \mathrm{~s}^{-1}$

- A planetary mass $M_{p}=3 \mathrm{M}_{\oplus}$ 
- A planetary orbital radius $a=5.2 \mathrm{AU}$

- The ratio of the specific heat at constant pressure to the specific heat at constant volume $\gamma=1.4$

- The mean molecular weight of the gas $\mu=2.3 \mathrm{~g} \mathrm{~mol}^{-1}$

- A surface density law given by:

$$
\Sigma(r)=200\left(\frac{r}{a}\right)^{-\sigma} \mathrm{g} \mathrm{cm}^{-2}
$$

with $\sigma=1 / 2$. This results in a value at $5.2 \mathrm{AU}$ that is roughly $30 \%$ above the value quoted for the Minimum Mass Solar Nebula ${ }^{21}$ (protoplanetary disk of minimum mass needed to form the Solar System).

The planetary potential is given by:

$$
\phi_{p}=-\frac{G M_{p}}{\left(r^{2}+\epsilon^{2}\right)^{1 / 2}},
$$

where $G$ is the gravitational constant, $r$ is the distance to the planet, and $\epsilon=2.5 \cdot 10^{-3} a$ a softening length used to avoid a divergence of the force in the planet vicinity.

\section{Comparison with a second hydrocode}

The numerical scheme of the FARGO3D code is in may aspects similar to that of the ZEUS code ${ }^{\sqrt{22}}$. There are, however, two notable differences: FARGO3D conserves (angular) momentum to machine accuracy, and it features orbital advection (which gives its name to the code through the acronym of Fast Advection in Rotating Gaseous Objects ${ }^{23}$ ). We have checked our results on the fiducial run using a version of the nested mesh code JUPITER ${ }^{24}$ that features a MUSCL-Hancock 
predictor step together with an exact adiabatic Riemann solver. As in FARGO3D, at each hydrodynamical time step, a radiative diffusion module based on flux limited diffusion in a two temperature approach is used to update the radiative and thermal energies. Solving for the radiative energy across the different mesh levels is done as in ${ }^{25}$. The heating torque that we find with this alternate code is within $25 \%$ of the heating torque found with FARGO3D on the same setup.

\section{Numerical setup}

Our mesh has resolution $1024 \times 512 \times 64$ in, respectively, azimuth, radius, and colatitude, with cell interfaces evenly spaced along each dimension. Since the energy is released in the immediate vicinity of the planet, the effect on the torque appears on a short timescale (typically the dynamical timescale), hence meaningful results can be obtained with short term simulations. We therefore run all our simulations over only 10 orbits. We have checked nevertheless with one setup (not presented here) that the heating torque is constant over a duration of 100 orbits, longer than the horseshoe libration timescale of our planets, from which we can discard the possibility that the heating torque could be a transient effect that would appear upon the insertion of the planet in the disc. For each given setup, we run in succession:

- A meridian $(r, \theta)$ two dimensional simulation to allow the disc to relax toward hydrostatic and radiative equilibrium.

- A first three dimensional calculation in which a planet is inserted without releasing energy in the ambient disc, which takes as initial condition the outcome of the previous run.

- A second three dimensional calculation, which also takes as initial condition the outcome of the first run, in which we insert a planet of same mass as introduced in the previous run but 
which this time is allowed to release energy.

Our mesh is designed so that the planet is at the intersection between cell interfaces in azimuth, radius and colatitude. The planet therefore lies at the centre of an eight cell cube. The energy it releases is added evenly to these eight cells at each time step. When integrating the torque exerted by the disc on the planet, we cut off the contribution of the cells that lie within half a Hill radius of the planet ${ }^{26}$. We find this cut off to have only a mild effect on the torque value.

The amount of energy released by the planet per unit time is:

$$
\dot{E}=\frac{G M \dot{M}}{R_{p}}=\frac{G M^{2}}{\tau R_{p}}
$$

where $\tau=M / \dot{M}$ is the planetary mass doubling time, and $R_{p}$ the physical radius of the planet, calculated assuming a density $\rho=3 \mathrm{~g} \mathrm{~cm}^{-3}$. The mass doubling time in our fiducial run is $\tau=10^{5}$ yrs. This equation applies to embryos that are not surrounded by a dense envelope, and it neglects the latent heat for vaporisation of the material (which is at most of order $10^{11} \mathrm{erg} / \mathrm{g}$, whereas $G M_{p} / R_{p} \sim 10^{12} \mathrm{erg} / \mathrm{g}$ for the fiducial run). We make no assumptions regarding the size of the solids that are impinging onto the core. Although we might expect a vigorous heating torque when an embryo is subjected to the potentially extremely effective "pebble accretion" mechanism, further progress is needed in assessing the altitude at which pebbles deposit their energy and the ultimate core luminosity. However, regardless of the core luminosity that might arise from pebble accretion, a minimal value for the luminosity is set by the accretion of planetesimals, which leads to mass doubling times typical of those considered in our analysis.

\section{Exploration of parameter space}

We have performed systematic explorations of the magnitude of the heating torque by varying one 
parameter at a time of our fiducial set up. Namely, we have varied the viscosity between $4 \cdot 10^{14}$ and $4 \cdot 10^{15} \mathrm{~cm}^{2} \mathrm{~s}^{-1}$, the surface density between 100 and $1000 \mathrm{~g} \mathrm{~cm}^{-2}$, the mass doubling time between $3 \cdot 10^{4}$ and $3 \cdot 10^{5}$ years, the opacity between 0.1 and $10 \mathrm{~cm}^{2} \mathrm{~g}^{-1}$, the planetary mass from one third of the Earth's mass to 7 Earth's masses, and the exponent $\sigma$ of the power law of the surface density, from -2 to 1.5 . These explorations show that the heating torque, normalised to the torque of the non-accreting case, depends only weakly on the surface density and viscosity. The dependency on $\sigma$ is as mentioned in the main text: the heating torque is found to scale with the distance between the planet and corotation. For the other parameters, we find the dependencies given in the Figure 4. The torque values reported in this figure are time-averages of the torque measured over orbits 5 to 10 of each calculation.

The heating torque depends strongly on the opacity and on the mass doubling time. At larger opacity, the energy radiated by the planet is trapped nearby it, hence the under-dense lobes are more pronounced and the net effect is stronger than for smaller opacity. When the mass doubling time decreases, the heating torque increases since $\dot{E}$ increases. We also find that the heating torque has a large efficiency over the mass interval $0.5-3 M_{\oplus}$. Its decline past this mass is compatible with the fact that the distance of the planet to corotation becomes an ever smaller fraction of the Hill sphere, so that the flow in the vicinity of the planet (and therefore the under-dense lobes) become more symmetric.

A final comment is warranted regarding the torque resulting from the angular momentum that is transferred to the planet by the material being accreted. We find it to be several orders of magnitude smaller than the heating torque, so it can be safely neglected. 


\section{Code availability}

The FARGO3D code is publicly available at the address http://fargo.in2p3.fr. This public version does not yet include the radiative transfer module, which will be made available once it has been fully documented. The JUPITER code is not publicly available at the present time.

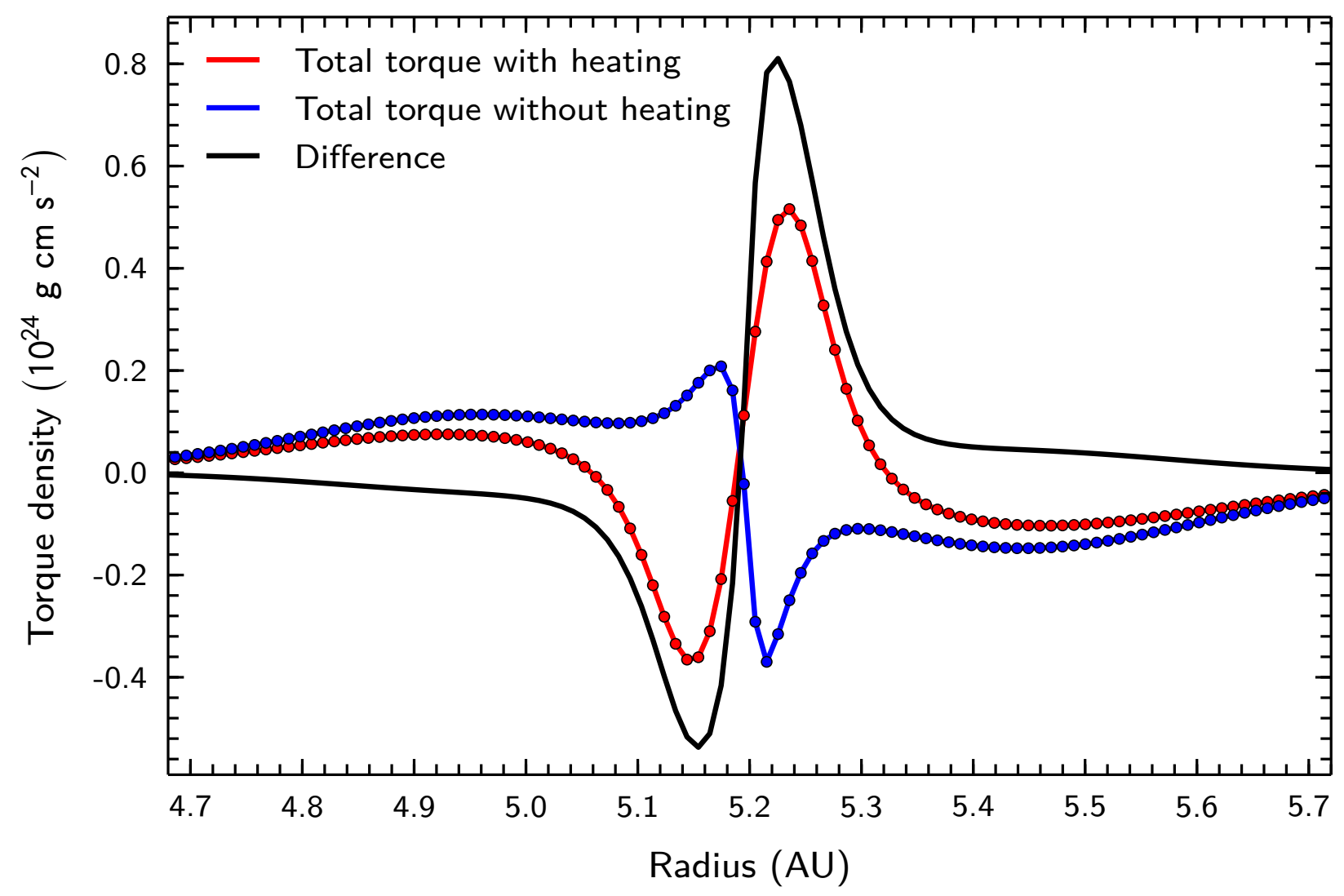

Figure 1: Comparison of the torques in the cases with and without heating. The blue curve shows the torque radial density (i.e. torque exerted by rings of unitary radial width upon the planet) in the non-heating case and the red curve when the heating is included. Their difference shows the heating torque density (black). This calculation corresponds to an embryo planet of 3 Earth masses which is located at $5.2 \mathrm{AU}$ from its central star. 


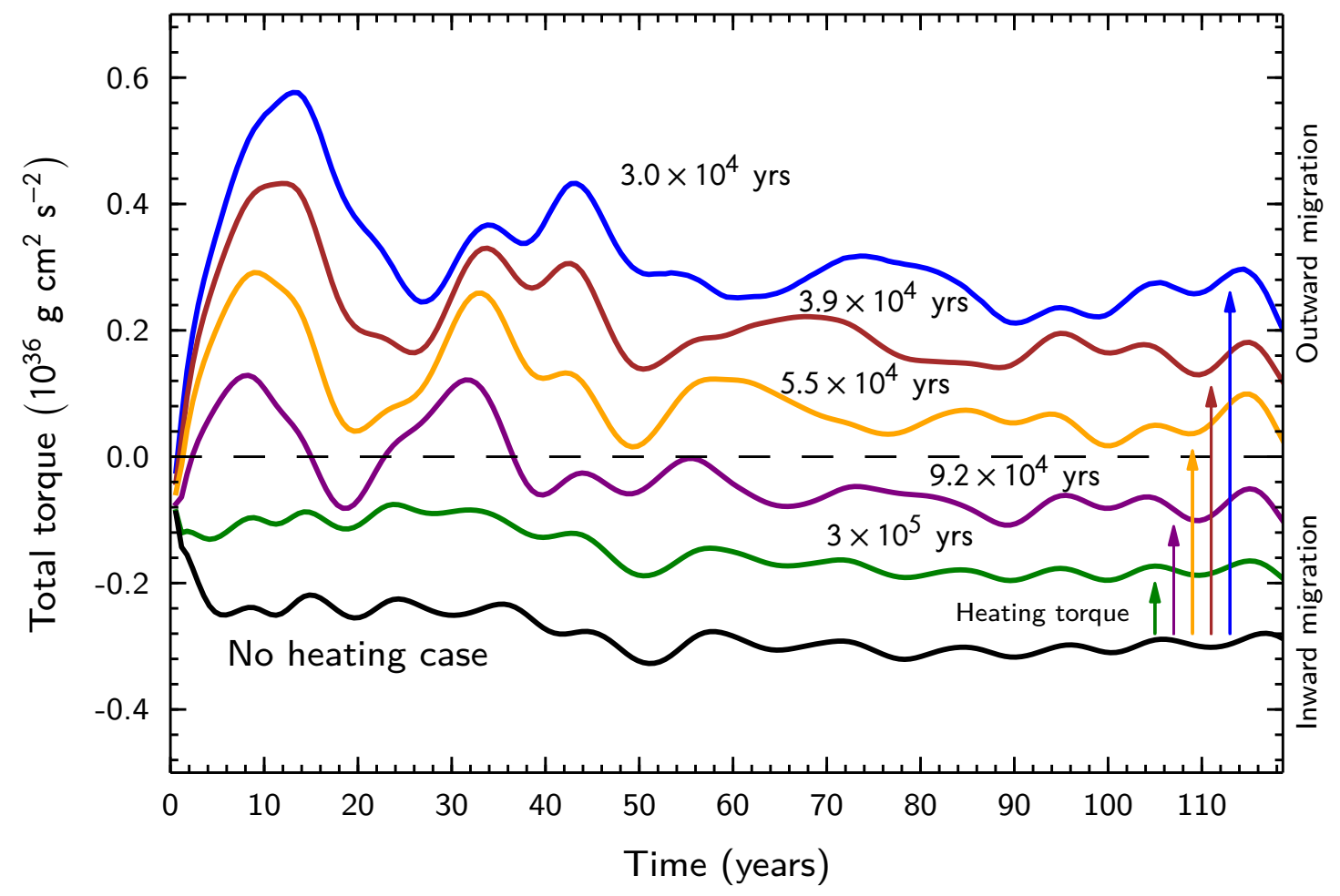

Figure 2: Heating torque for different growth time scales. The curves are labelled with the planetary mass doubling time (i.e. the time it takes the accreting planet to double its mass) and show the torque exerted on a 3 Earth masses embryo planet over the first 118 years of our calculation (10 orbits). With low or no heating, the planet migrates inward while for larger rates (mass doubling time shorter than 92 thousand years) it migrates outward. The dashed line corresponds to no migration. The vertical arrows show the magnitude of the heating torque. 


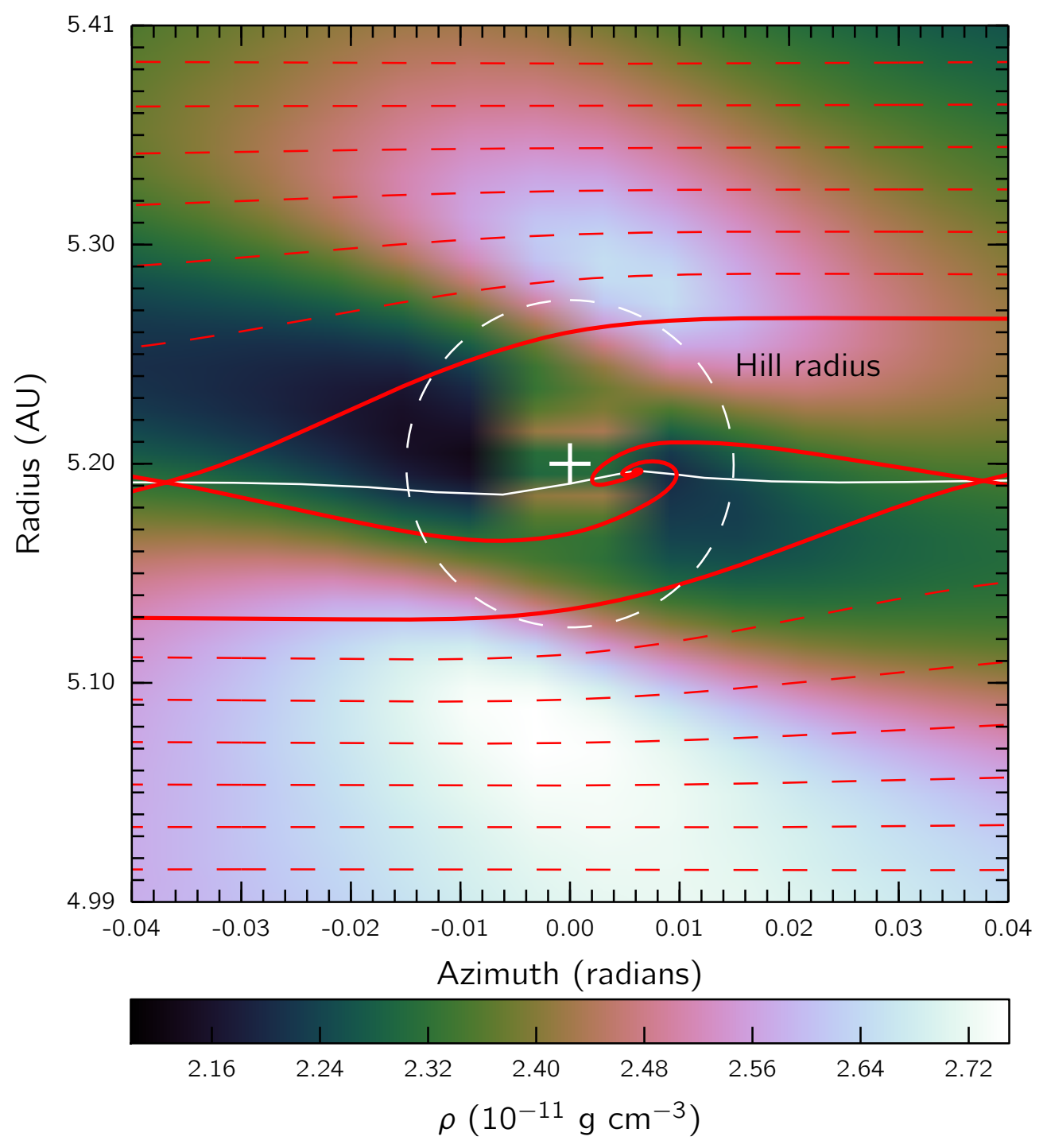

Figure 3: Density in the vicinity of an irradiating embryo. This equatorial slice of the mass density shows two low density lobes on each side of the planet (cross). The more apparent one (left) gives rise to the positive torque. Such lobes are absent for a non-radiating embryo. The dashed circle shows the planetary Hill radius. Streamlines are in red showing separatrices of the coorbital region (bold) and paths of material further from the planet (dashed). The white nearly horizontal curve shows corotation, i.e. the place where the material is at rest in the planet frame. 


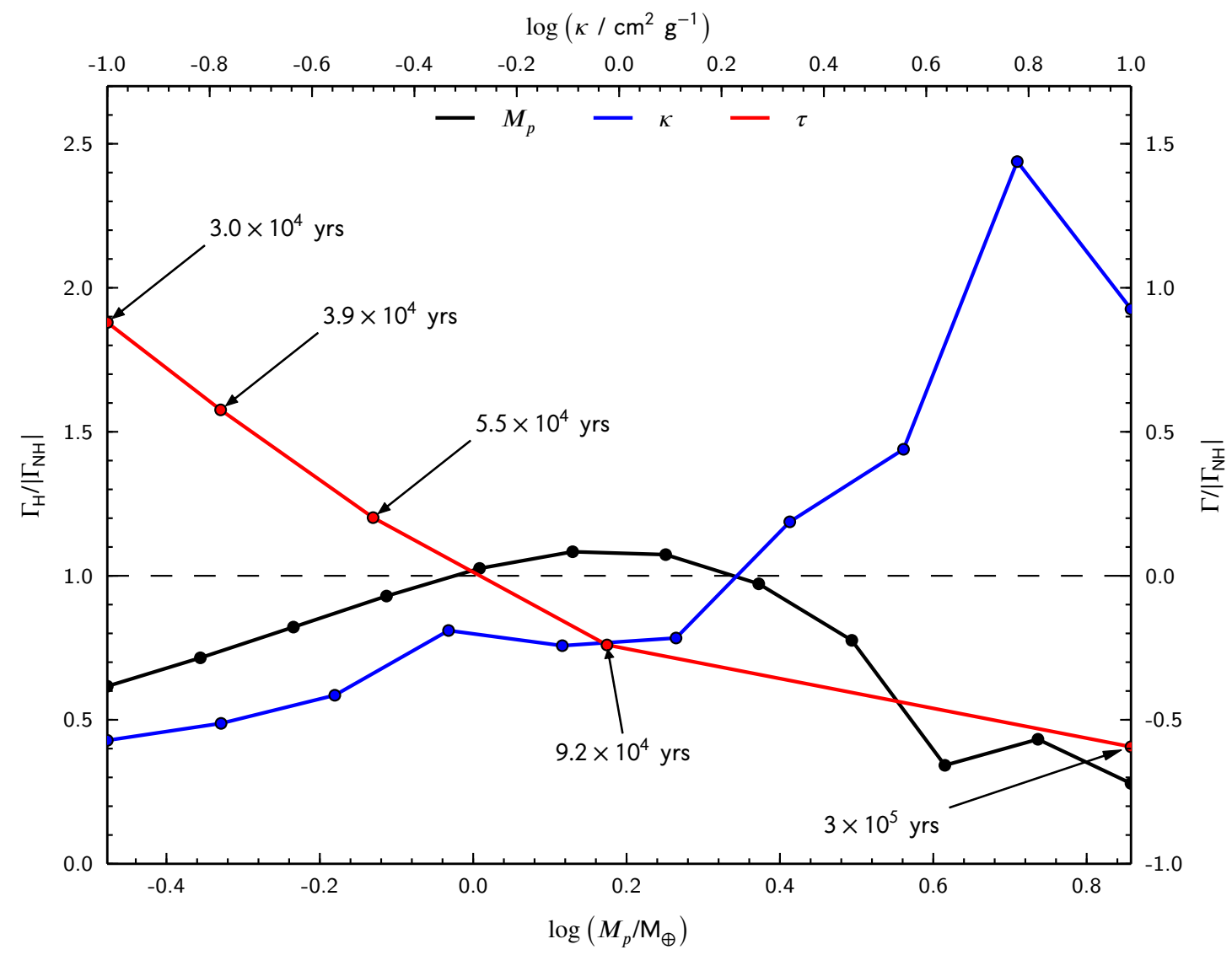

Figure 4: Exploration of the parameter space. Heating torque $\Gamma_{\mathrm{H}}$ normalised to the absolute value of the torque of the non-accreting case $\left|\Gamma_{\mathrm{NH}}\right|$, as a function of embryo mass $M_{p}$, opacity $\kappa$ and mass doubling time $\tau$. Whenever one parameter is varying, others have the value of the fiducial run. Mass doubling times are given in units of years and show that a positive torque results for $\tau \lesssim 60,000$ years. The right axis shows the total torque $\Gamma=\Gamma_{\mathrm{H}}+\Gamma_{\mathrm{NH}}$, also normalised to $\left|\Gamma_{\mathrm{NH}}\right|$. The horizontal dashed line corresponds to no migration. 


\section{References}

[20] J. P. Ramsey and C. P. Dullemond. Radiation hydrodynamics including irradiation and adaptive mesh refinement with AZEuS. I. Methods. Preprint at http://arxiv.org/abs/1409.3011, 2014.

[21] C. Hayashi, K. Nakazawa, and Y. Nakagawa. Formation of the solar system. In D. C. Black and M. S. Matthews, editors, Protostars and Planets II, pages 1100-1153, 1985.

[22] J. M. Stone and M. L. Norman. ZEUS-2D: A radiation magnetohydrodynamics code for astrophysical flows in two space dimensions. I - The hydrodynamic algorithms and tests. Astrophys. J. Suppl. Ser., 80:753-790, 1992.

[23] F. Masset. FARGO: A fast eulerian transport algorithm for differentially rotating disks. A\&AS, 141:165-173, 2000.

[24] J. Szulágyi, A. Morbidelli, A. Crida, and F. Masset. Accretion of Jupiter-mass Planets in the Limit of Vanishing Viscosity. Astrophys. J., 782:65, 2014.

[25] B. Commerçon, V. Debout, and R. Teyssier. A fast, robust, and simple implicit method for adaptive time-stepping on adaptive mesh-refinement grids. Astron. Astrophys., 563:A11, 2014.

[26] A. Crida, C. Baruteau, W. Kley, and F. Masset. The dynamical role of the circumplanetary disc in planetary migration. Astron. Astrophys., 502:679-693, 2009. 